\title{
The Artificial University: Decision Support for Universities in the COVID-19 Era
}

\author{
Wesley J. Wildman $\mathbb{D}^{1,2,3}$ Saikou Y. Diallo ${ }^{1 D},{ }^{1,4}$ George Hodulik ${ }^{1},{ }^{1}$ Andrew Page $\mathbb{D}^{1,5}$ \\ Andreas Tolk $\mathbb{D}^{1,}{ }^{1,6}$ and Neha Gondal $\mathbb{D}^{1,2,7}$ \\ ${ }^{1}$ Center for Mind and Culture, 566 Commonwealth Ave, Suite M-2, Boston, MA 02215, USA \\ ${ }^{2}$ Faculty of Computational and Data Sciences, Boston University, 111 Cummington St, Boston, MA 02215, USA \\ ${ }^{3}$ School of Theology, Boston University, 745 Commonwealth Ave, Boston, MA 02215, USA \\ ${ }^{4}$ Virginia Modeling, Analysis \& Simulation Center, Old Dominion University, 1030 University Blvd., Suffolk, VA 23435, USA \\ ${ }^{5}$ Translational Health Research Institute, Western Sydney University, Building 3, David Pilgrim Ave., Campbelltown, \\ NSW 2560, Australia \\ ${ }^{6}$ Modeling, Simulation, Experimentation, and Analytics, The MITRE Corporation, 1001 Research Park Blvd. \#220, \\ Charlottesville, VA 22911, USA \\ ${ }^{7}$ Department of Sociology, Boston University, 96 Cummington St, Boston, MA 02215, USA
}

Correspondence should be addressed to Wesley J. Wildman; wwildman@bu.edu

Received 26 July 2020; Revised 24 November 2020; Accepted 10 December 2020; Published 30 December 2020

Academic Editor: xiaoke xu

Copyright ( $\odot 2020$ Wesley J. Wildman et al. This is an open access article distributed under the Creative Commons Attribution License, which permits unrestricted use, distribution, and reproduction in any medium, provided the original work is properly cited.

\begin{abstract}
Operating universities under pandemic conditions is a complex undertaking. The Artificial University (TAU) responds to this need. TAU is a configurable, open-source computer simulation of a university using a contact network based on publicly available information about university classes, residences, and activities. This study evaluates health outcomes for an array of interventions and testing protocols in an artificial university of 6,500 students, faculty, and staff. Findings suggest that physical distancing and centralized contact tracing are most effective at reducing infections, but there is a tipping point for compliance below which physical distancing is less effective. If student compliance is anything short of high, it helps to have separate buildings for quarantining infected students, thereby gracefully increasing compliance. Hybrid in-person and online classes and closing fitness centers do not significantly change cumulative infections but do significantly decrease the number of the infected at any given time, indicating strategies for "flattening the curve" to protect limited resources. Supplementing physical distancing with centralized contact tracing decreases infected individuals by an additional 14\%; boosting frequency of testing for student-facing staff yields a further $7 \%$ decrease. A trade-off exists between increasing the sheer number of infection tests and targeting testing for key nodes in the contact network (i.e., student-facing staff). There are significant advantages to getting and acting on test results quickly. The costs and benefits to universities of these findings are discussed. Artificial universities can be an important decision support tool for universities, generating useful policy insights into the challenges of operating universities under pandemic conditions.
\end{abstract}

\section{Introduction: The Crying Need for Insight}

Operating institutions of higher education under SARSCoV-2 pandemic conditions is a perilous, complex, and expensive undertaking. Simple simulations of epidemiological models can be adapted to allow university administrators to test combinations of interventions but such models typically neglect the human factors (e.g., social networks and multiple dimensions of compliance) that heavily influence whether interventions fail or succeed. Bespoke policy simulations incorporating confidential data about students and staff are prohibitive for many schools in terms of their cost and the expertise needed to build them, and they cannot be shared, duplicating effort. Dashboards offering generic advice do not take account of the facts that universities vary widely in what interventions are politically 
feasible (e.g., shutting down football is unthinkable in some places, and centralized contact tracing is too controversial in others) or financially achievable (a massive testing, tracing, and quarantine regimen can be prohibitively expensive in terms of materials, space, and personnel). Most dashboards are using available data to support smart extrapolation into the future, while such data are typically not available for universities. Moreover, universities are diverse in terms of what counts as success, and thus they would naturally apply different metrics to evaluate the effectiveness of combinations of interventions (e.g., minimizing infections, or optimizing good outcomes versus bad outcomes). There is an urgent need for flexible and timely policy modeling that can be applied in a host of different institutional contexts.

To respond to this need, the Human Simulation Group created The Artificial University (TAU). TAU is a flexible computer simulation of a university based solely on publicly available web-scraped data about typical class schedules, residence and dining arrangements, age distributions of faculty and staff, on-campus and off-campus activities, gyms, clubs, and commuting. TAU has a threefold configuration process to render the artificial university a close match to any real-world university (Figure 1).

First, TAU can be configured to match university characteristics. TAU handles schools of any size and composition, both two-year and four-year colleges without graduate students and research universities with graduate students. The simulation takes account of faculty and staff age distributions, class schedules, a complex variety of oncampus and off-campus residences with diverse bathroomsharing arrangements, campus dining halls, specialized quarantine buildings and floors, on-campus gyms and clubs, on-campus sports and public events, and off-campus contacts and commutes. The simulation-based approach permits the application of various knowledge-based prognostication methods and is not limited to smart extrapolation of data.

Second, TAU can be configured to match the range of feasible interventions for a given university setting. Interventions in TAU include allowing or closing gyms, sports, student clubs, and on-campus events; hybrid classes to dedensify classrooms while maintaining live education both in-person and online; testing regimens of varying capacities and reliabilities, both for infection and antibodies, and with varying delays in getting results as well as the possibility of boosting the frequency of infection testing based on antibody testing results, or on whether staff have student-facing jobs; contact tracing of varying intensities, from anonymous apps to centralized tracking by university administrators; and varying intensities of quarantine from self-isolation to placing symptomatic people in quarantine buildings supported by staff. TAU also takes account of compliance with physical distancing requirements, with reporting symptoms to a contact-tracing app, and with self-isolation expectations. These critical human factors can vitiate the effectiveness of interventions.

Third, TAU can be configured to respond to the valueladen perspectives of universities, which yield very different metrics for assessing whether a combination of interventions is successful. Universities might emphasize the danger of COVID-19 for vulnerable people, including older faculty and staff and people of all ages with preexisting conditions, in which case they will seek to maximize the number of people never infected. Some might prefer a hybrid metric that optimizes the difference between positive outcomes (never infected or recovered) and negative outcomes (deaths or reinfections). Still others might want to take account equity and distributive justice concerns and all will need to account for financial realities. The outcome metrics matter, in the specific sense that they lead to different priorities for intervention arrays. TAU supports all viewpoints on what is an intricately complex, multiattribute, multivalue decision problem. The modeling process itself helps to make problems and solution methods tangible by describing them in the common language of the simulation, so that trade-offs and compromises become clearer and unintended consequences in other value domains may be avoided.

\section{Materials and Methods}

2.1. Computational Simulation Design. The computational policy simulation that powers TAU is an agent-based epidemiological model (just as for [1]). Each individual agent, whether student, faculty, or staff, moves between states of being susceptible, infected, recovered, and resusceptible, depending in part on biological factors. (It should be noted that these are individual agent states used to trace whether an agent is likely to infect another agent. TAU is not a population-compartmental model, as often used in epidemiology simulation.) Since there have been confirmed reinfections (i.e., the virus causing the second infection is genetically different from the virus causing the first), it is important to allow for the possibility that periods of immunity are short.

TAU is not a spatial model; it is a contact-network model. Network links are the pathways for possible meetings with infectious people. Thus, two agent nodes are linked when there is physical contact and therefore the possibility of infection, indicated by being in class together, living together in a dorm, or both going to the gym or a university event. Links are weighted according to the likelihood of infection. Because universities have a rhythmic schedule over the course of a week, the network is static. Interventions modify network links, reducing the likelihood of meetings, and therefore also of infection. For example, physical distancing, mask-wearing, closing gyms, and dedensifying classrooms by using a hybrid in-person and remote teaching system reduce the probability of infection and thus lighten link weights, or sometimes eliminate links altogether. The higher the levels of compliance, the lower the probabilities of infection. Additional networks can be added, such as family, friends, or other social connections, but, for these networks, usually no data exist on the university level. Further details about TAU's design-including complete documentation of entities, state variables, time scales, networks, network link types, process scheduling, parameters, and initialization-are provided in the online model documentation at https://github.com/centerformindandculture/ TheArtificialUniversity. 


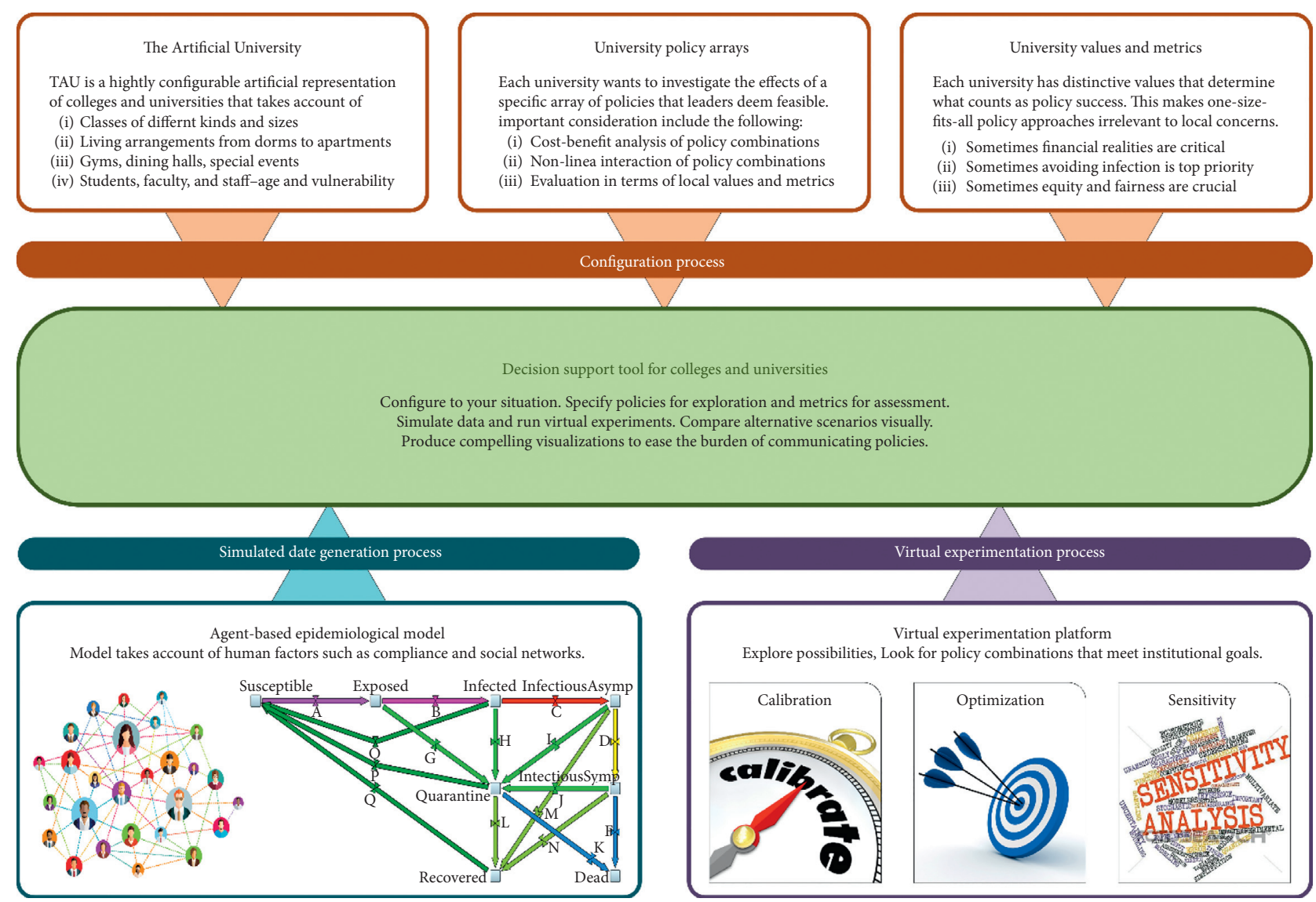

Figure 1: Diagram of TAU, a decision support tool for colleges and universities. The threefold configuration process is at the top, the simulation and social network feeding TAU is at the bottom left, and the virtual experimental platform is at the bottom right.

Note that the networks employed in this model are based on copresence (approximating contact on the basis of occupying spaces at the same time) rather than relationship data (social connections such as friendship and familial ties). Copresence data addresses both modeling and practical concerns. With respect to modeling the spread of SARS$\mathrm{CoV}-2$, sustained copresence (such as seated in close proximity in a classroom) or joint living circumstances (such as suitemates or floor-mates with shared bathroom facilities) are crucial vectors of transmission. Practically speaking, relationship data on ties such as friendship can be highly sensitive to social contexts and, consequently, difficult to generalize. Moreover, relational data are resource intensive whereas copresence data are easier to assume based on the distribution of events and living conditions.

\subsection{Design of Experiments: Exploratory Analysis. TAU is} highly stochastic and thus we built a large dataset by sweeping the parameter space with 30 replications for each combination of parameter settings, seeking $95 \%$ confidence in the outcome variables. This technique of exploratory analysis is often used in areas of deep uncertainty (e.g., [2]). It surfaces emergent behavior and changes in the metabehavior of the system over the solution space, including tipping points defining the borders of behavior regimes. By surfacing such information and being clear about assumptions, TAU promotes thinking deeply into a complex situation rather than delivering straightforward answers to important questions (see [3]). TAU's capacity to promote deeper understanding of a complex management problem is particularly important given that real-world data for universities does not exist to validate epidemiological models in a quantitative way. Prominent university closures in September 2020 confirm that failures of compliance can comprehensively undermine a COVID-management plan, and this is a useful qualitative confirmation of TAU's finding that compliance is the single most important explanatory factor, but quantitative validation must wait for new kinds of datasets to emerge.

We present an evaluation of an array of interventions using health-outcome metrics (e.g., minimizing infections) for two artificial universities, a four-year college and a research university (see Table 1). Most configurable aspects of the universities (e.g., residence arrangements, commuting, class schedules, and activities) are linearly scaled with the population so that the main difference between the two universities is size.

Results for the two artificial universities are similar, so we report here on the smaller university of 6,500 people unless otherwise noted. We do not report on different configurations for universities of the same type and size (e.g., 
TABLE 1: Basic characteristics of the two types of universities tested.

\begin{tabular}{|c|c|c|}
\hline Characteristic & Four-year college & Large research university \\
\hline $\begin{array}{l}\text { Size and } \\
\text { composition }\end{array}$ & 6,500 students, faculty, and staff, no graduate students & 37,200 students, graduate students, faculty, and staff \\
\hline $\begin{array}{l}\text { Student-facing } \\
\text { staff }\end{array}$ & $\begin{array}{l}10 \% \text { of staff are student-facing, with } 100 \text { interactions per } \\
\text { day with students }\end{array}$ & $\begin{array}{l}10 \% \text { of staff are student-facing, with } 200 \text { interactions per } \\
\text { day with students }\end{array}$ \\
\hline Testing & $\begin{array}{c}\text { Maximum 1,000 infection tests and maximum } 100 \\
\text { antibody tests per day }\end{array}$ & $\begin{array}{c}\text { Maximum 5,000 infection tests and maximum } 500 \\
\text { antibody tests per day }\end{array}$ \\
\hline $\begin{array}{l}\text { Living } \\
\text { arrangements }\end{array}$ & $\begin{array}{l}3 \text { dining halls serving a mix of large dorms sharing } \\
\text { bathrooms and apartment style living for on-campus } \\
\text { residents; others commute from apartments and homes }\end{array}$ & $\begin{array}{l}15 \text { dining halls serving a mix of large dorms sharing } \\
\text { bathrooms and apartment style living for on-campus } \\
\text { residents; others commute from apartments and homes; } \\
\text { same ratios as for the four-year college }\end{array}$ \\
\hline
\end{tabular}

Note. Most configurable aspects of the universities are scaled with population size in a nearly linear fashion.

mostly commuter students versus mostly on-campus residents). TAU surfaces surprising and helpful information about interventions and tipping points for their effectiveness, which should be useful to university planners.

We varied parameters related to specific interventions. Using a Latin-hypercube-sampling method, we identified 500 parameter combinations to explore in a parameter sweep. We ran each parameter combination 30 times or until $95 \%$ confidence was achieved for three output metrics: the number of people never infected, the total number of infections (including reinfections), and the hybrid metric that measures the difference between positive outcomes (never infected or recovered) and negative outcomes (deaths or reinfections). The resulting 15,000 runs took several days on a machine with a maximum availability of 128 cores and 1 TB RAM. We then ran analyses of the simulated dataset through the statistical package $R$, beginning with a sensitivity analysis of intervention parameters and passing to more specific tests.

We set epidemiological parameters based on reviews of epidemiological and virology literature. For the 6,500-person university reported on here, we fixed a lot of other parameters using findings from scraped data: staff-facultystudent ratios, proportion of students in on-campus dorms, distribution of types of dorms and the number of students sharing bathrooms, distributions of dining halls, frequency of campus events, clubs and sports, class schedules, and offcampus events.

TAU was developed in AnyLogic version 8.5.2. The opensource model and documentation are available at https:// github.com/centerformindandculture/TheArtificialUniversity. A dashboard for TAU is presented at http://mindandculture. org/projects/modeling-social-systems/vivid/vivid-dashboard/. The dashboard facilitates the exploration of the impact of a variety of specific interventions on the university population using two different health-outcome metrics.

\section{Results}

3.1. Identifying Which Interventions Have the Greatest Impact. The single most important intervention is high compliance with physical distancing. In TAU, physical distancing reduces the probability of an infection through a network link and corresponds in the real world to wearing masks and keeping physically separate from others. Using the "minimize infections" metric with the adjusted- $R^{2}$ test, high compliance with physical distancing explains $70 \%$ of the variance (Figure 2).

Adding the variant of contract tracing that involves centralized tracking and strong follow-up to ensure selfisolation brings the total variance explained to $83 \%$. Adding a policy to boost testing frequency of staff with studentfacing jobs (e.g., people working in dining halls, cleaning student areas, and meeting intensively with students) further increases the variance explained to $86 \%$.

Note that the adjusted $-R^{2}$ test incorporates a penalty for adding additional factors into the regression, so there is a convergence effect as more interventions are included. It follows that the less important interventions in the hierarchy of Figure 2 could still be important when considered alone or in combination with high compliance with physical distancing. To explore this possibility, the TAU dashboard (http://mindandculture.org/projects/modeling-social-systems/ vivid/vivid-dashboard/) allows users to visualize the projected health effects of varying individual interventions.

If we assume student compliance with social distancing will not be better than 50\% (probably a reasonable assumption), do the other interventions still rank order the same way? To answer this question, we fixed faculty and staff compliance of all kinds at a high level and fixed student compliance of all kinds at 50\% (save for compliance with forced quarantine in a separate building for infected students, which was fixed to high, and the intervention itself was allowed to be on or off). Under those circumstances, five factors explain the bulk of variance in the "number never infected" metric. The top three hit in the same order as before (after compliance factors are eliminated), though the variance explained is lower, a reminder of how important compliance is as follows:

(1) Centrally monitor contact tracing $\left(\operatorname{adj}-R^{2}=0.64\right)$

(2) Add: boost testing for student-facing staff $\left(\operatorname{adj}-R^{2}=0.75\right)$

(3) Add: hybrid classes to dedensify rooms $\left(\operatorname{adj}-R^{2}=0.78\right)$

The next two factors are as follows: 


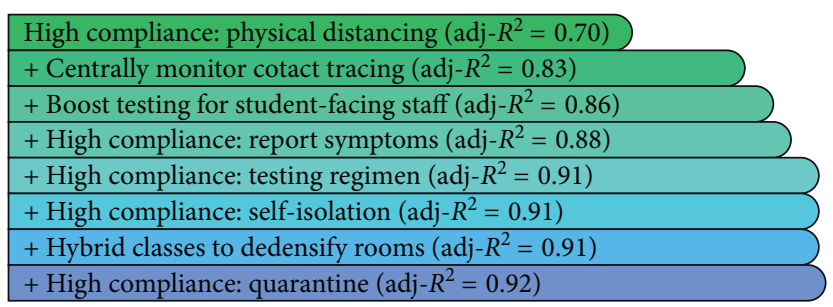

Figure 2: Regression-subset diagram for TAU using the "minimize infections" metric on a university of 6,500 people. The single most important intervention is on the top row, explaining $70 \%$ of the variance in the outcome metric according to the adjusted- $R^{2}$ test. The second row shows the effect of adding the second most important explanatory factor into the regression (adjusted- $R^{2}$ lifts to 0.83 ). As each new factor is added, there are marginal gains in the adjusted- $R^{2}$ value.

(4) Add: forcing quarantine of infected students in a separate building, which improves compliance $\left(\operatorname{adj}-R^{2}=0.78\right)$

(5) Add: deprioritizing infection testing for people who test positive for antibodies, freeing tests for others $\left(\operatorname{adj}-R^{2}=0.78\right)$

Change the metric to "number infected (including reinfections)" and the last two factors switch order.

We also asked what would happen if centralized contact tracing was not used, thereby addressing a big concern for colleges and universities having students, faculty, or staff who are hyperconscious about privacy. In that case, interventions line up in a similar way, with a slightly different order, and much lower adj- $R^{2}$ values. Note the appearance of a new factor in fourth place, increasing testing frequency for older faculty and staff:

(1) Boost infection testing for student-facing staff (adj$R^{2}=0.31$ )

(2) Add: forcing quarantine of infected students in a separate building, which improves compliance $\left(\operatorname{adj}-R^{2}=0.42\right)$

(3) Add: hybrid classes to dedensify rooms $\left(\operatorname{adj}-R^{2}=0.50\right)$

(4) Add: boost infection testing for older faculty and staff $\left(\operatorname{adj}-R^{2}=0.52\right)$

These results drive home the importance of high student compliance with every aspect of the university intervention program, the impact of centralized contact tracing, and the value of "gracefully forcing" infected students into highcompliance mode by using a separate quarantine space.

It is also important to notice which interventions had relatively lower effects on health outcomes. They include the following:

Closing fitness centers

Closing student groups and clubs

Canceling large sport events

Canceling large events over a threshold size

Boosting testing frequency for people with health vulnerabilities

Lengthen contact-tracing history from 7 days to 14 days
In the large research university, TAU produced a similar but not identical list of factors accounting for variance in health outcomes. Here are the top six factors (note the higher values for adj- $R^{2}$ ):

(1) High compliance: physical distancing $\left(\operatorname{adj}-R^{2}=0.79\right)$

(2) Add: boost infection testing for student-facing staff $\left(\operatorname{adj}-R^{2}=0.87\right)$

(3) Add: boost infection testing for older faculty and staff $\left(\operatorname{adj}-R^{2}=0.90\right)$

(4) Add: centrally monitor contact tracing $\left(\operatorname{adj}-R^{2}=0.93\right)$

(5) Add: high compliance: testing regimen $\left(\right.$ adj- $\left.R^{2}=0.94\right)$

(6) Add: high compliance: report symptoms (adj- $\left.R^{2}=0.95\right)$.

3.2. Hybrid Class Structures. With universities facing countless class-action lawsuits alleging failure to provide the promised educational experience due to a switch early in 2020 to remote education, being able to provide a safe, highquality education that is live and in-person is a priority for university administrators. One way to achieve this is a hybrid system that conducts live classes with two groups simultaneously, one in person and the other remote. This approach dedensifies classrooms while remotely including vulnerable students and students in quarantine. The hybrid system also permits international students who may not be allowed into the country to continue their education.

In TAU, hybrid classrooms work by splitting classes that meet three times a week into three equal-sized, nonoverlapping platoons of students who attend class face to face one day per week and attend remotely for the other two days-all occurring in a room capable of holding the entire class at once. Classes that meet twice per week use two platoons of students, and long classes that meet once per week have two platoons that alternate weeks attending in person. Figure 3 assesses the effectiveness of this intervention strategy. The number never infected by the end of the simulation (Figure 3(a)) is not significantly improved with hybrid classes, but the maximum number infected at any given time (Figure 3(b)) is significantly reduced (by about 30\%). It follows that university administrators should not expect 


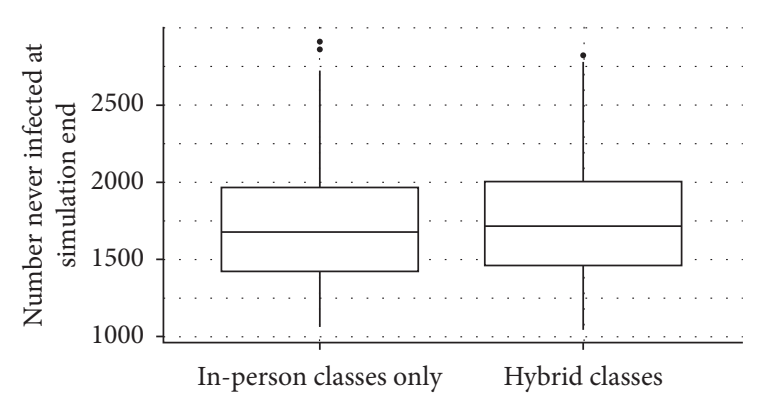

(a)

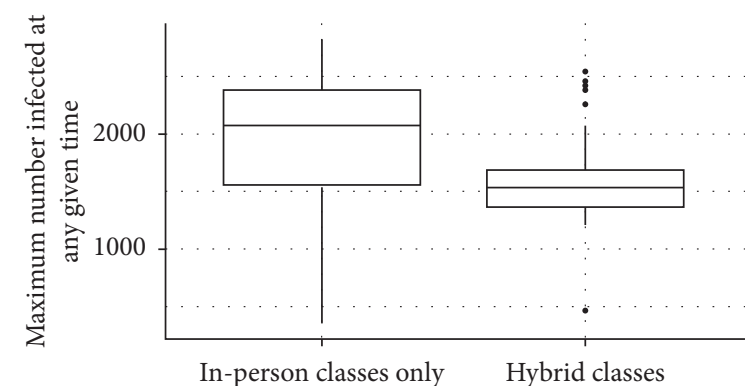

(b)

FIgURE 3: Box plots illustrating the effect of introducing hybrid university classrooms. The left image in (a) shows that there is little difference in the number never infected, while the right image in (b) shows that the maximum number infected at any given time is significantly lower with a hybrid class system. The lower and upper hinges correspond to the first and third quartiles (the $25^{\text {th }}$ and $75^{\text {th }}$ percentiles). The upper whisker extends from the hinge to the largest value no further than $1.5 *$ IQR from the hinge (where IQR is the interquartile range or distance between the first and third quartiles); similarly for the lower whisker. Data beyond the end of the whiskers are outlying points and are plotted individually.

hybrid classes to dramatically lower overall infections, but they can reasonably expect to "flatten the curve" by slowing down the rate of infection.

We hypothesize that platoons in classrooms have a limited impact on increasing the total number of people never infected because most students still live in on-campus housing. It may be possible to select platoons in such a way that they correspond to campus regions, and the resulting spatial compartmentalization might help to confine the spread of any outbreaks. But this is difficult, probably prohibitively difficult, to implement in a real-world class schedule with different class frequencies and compositions, so TAU does not evaluate this possibility.

Hospital emergency departments critically require slowing the rate of infection to avoid overrunning resources. Depending on their policies, universities can face a similar challenge, particularly if they set aside a certain number of buildings with bathroom-equipped rooms for people with symptoms who are quarantined. Hybrid classes can flatten the curve to avoid overrunning those vital and limited resources, even if they only marginally increase the number of people who are never infected. Much the same applies to closing fitness centers, which ought to be vectors of transmission through surfaces and heavy breathing. Such interventions do not appreciably lower the total number eventually infected but they do slow the rate of infection. If flattening the curve is important, then these policies make sense.

\subsection{Tipping Points for Compliance with Physical-Distancing Guidelines. Human beings exhibit a rich variety of per- sonalities, convictions, ideologies, and degrees of proso- ciality, resulting in varying willingness to comply with physical-distancing guidelines. Physical distancing is the single most important intervention for optimizing social- health metrics, but it requires sustained compliance, which young adults in particular often find trying. Consequently, there is an important question about how much of a dif- ference physical distancing really makes.}

Figure 4 shows the number of infections at simulation end against the degree of compliance with physical distancing, over all parameter combinations. There is a tipping point around 0.6 (in the simulation, this means $60 \%$ probability of being compliant). While the tipping point varies slightly depending on the precise parameter set in play, the response curve always has this shape and indicates that university administrators should expect increased gains with high compliance but few gains with low compliance. That is, if physical distancing is not done well, there is little point in doing it at all. It follows that universities should make physical distancing as easy as possible with masks, spacing marked for queues, and one-way people traffic where possible. University communities (including students themselves) need to promote the benefits of compliance with physical distancing guidelines-particularly with younger people in their community.

\subsection{Supplementing Physical Distancing with Testing and} Contact Tracing. On the "minimize infections" metric, the next best interventions (after high compliance with physical distancing) are rigorous contact-tracing and flexible testing strategies. How much of a difference do they make on minimizing the number of infections? Findings from TAU suggest the following:

Central tracking (i.e.,, centralized information about contact tracing, followed by enforcement of isolation among traced individuals) increases the number of people never infected by $14 \%$ (this is all of the policy variations with central tracking compared to all of the policy variations without central tracking)

Testing student-facing staff members more often additionally increases the number of people never infected by $7.6 \%$

High compliance with contact-tracing demands, which means reporting when COVID-19 symptoms are experienced or when a test result indicates infection, further increases the number of people never infected by $4.5 \%$ 


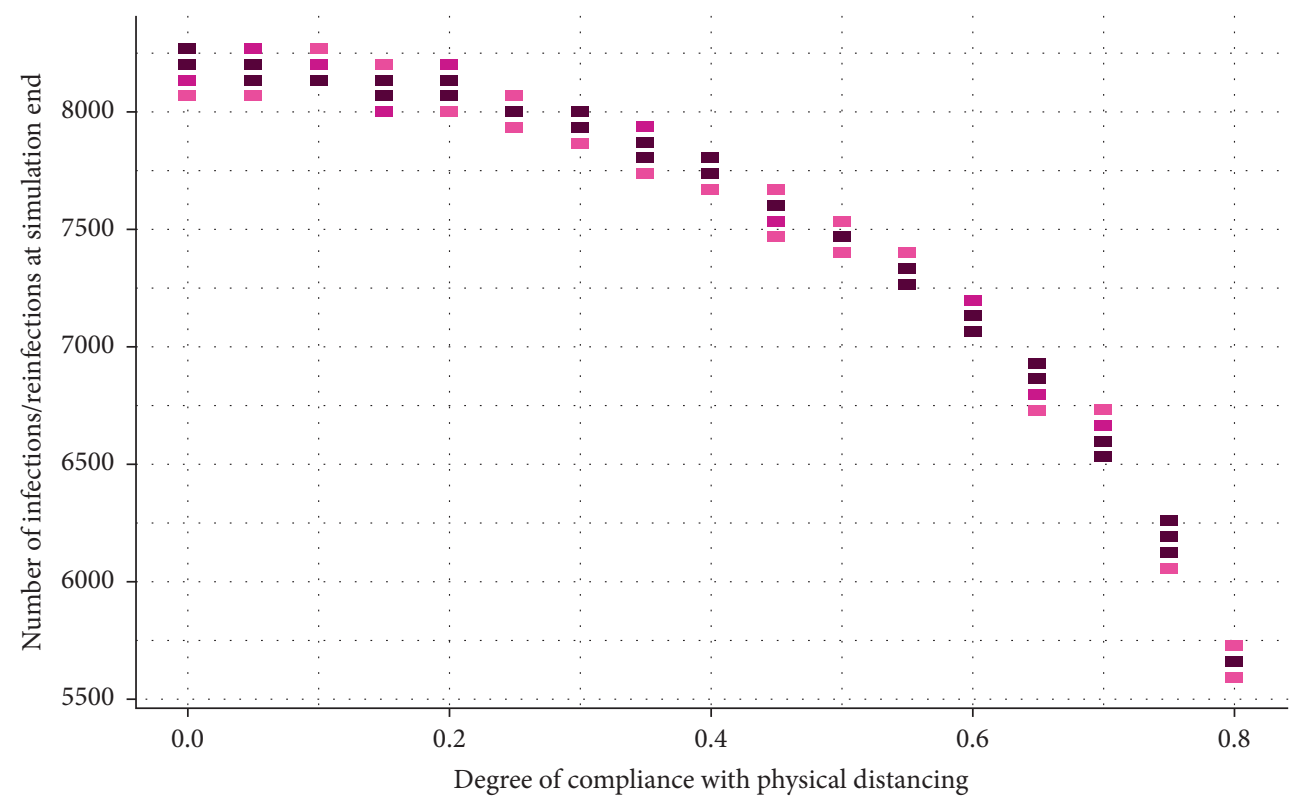

FIGURE 4: The number of infections against the degree of compliance with physical-distancing requirements (including mask-wearing). Note that the "number of infections" metric includes reinfections, so the score can exceed the number of people in the university $(6,500)$. Numerous runs are binned and the color indicates clustering of runs, with darker colors corresponding to a greater number of runs.

Increasing the frequency of testing staff with studentfacing jobs poses an important cost-benefit analysis puzzle because testing is expensive and frequent testing is proportionally more costly. We asked TAU how increasing the frequency of testing for staff-with student-facing jobs affects the number of infections (Figure 5). There is an inflection point around a boost of 4 times, taking account of all parameter combinations, suggesting that there is little gain from testing student-facing staff more than about four times more frequently than others.

3.5. Optimizing Testing. TAU examines the scenario where there is no infection testing alongside 16 infection-testing strategies, incorporating an antibody testing strategy. The 16 infection-testing strategies depend on the following:

Decreasing infection-testing frequency after a positive antibody test (YES if antibody testing is activated, and NO otherwise; positive antibody tests are not possible until at least four weeks after recovery)

Boosting infection-testing frequency for student-facing staff (YES/NO)

Boosting infection-testing frequency for people with health vulnerabilities (YES/NO)

Boosting infection-testing frequency for older people on campus (YES/NO)

There is a delay in receiving infection test results, varying from 24 to 72 hours. Both types of tests vary in cost with the more expensive being more accurate, and there is an economy of scale whereby testing more yields lower per-test cost. TAU is supplied with a fixed number of tests of both types per day, which yields a fixed cost for testing with a specified cost uncertainty (important for universities standing up internal testing facilities where costs are uncertain). TAU is also supplied with measures of accuracy (the likelihood of false positives and false negatives).

Analysis suggests that any testing regimen is far better than none. For infection testing, the most important factor is the number of tests per week, followed by boosting testing frequency for high-contact nodes in the physical contact network. Other testing options produce marginal returns by comparison. Figure 6 shows the situation in the artificial university after 120 days for six different testing configurations. The horizontal axis shows the average weekly testing frequency for studentfacing staff members, the vertical axis shows the number never infected at the simulation end, and each curve shows the number of tests per week. The shape of these curves shows that there is a trade-off between these two considerations such that testing key people more often can be more cost-effective than simply increasing the number of tests.

Figure 7 shows the situation in the artificial university after 120 days for three different testing configurations. The horizontal axis shows the number of viral tests per day, the vertical axis shows the number never infected, the color indicates the testing frequency boost for student-facing staff, and the shape indicates the delay in receiving testing results ( 24 hours or 72 hours). Getting infection-test results quickly ( 24 hours rather than 72 hours) makes a significant difference.

3.6. Downstream Consequences of Traced Isolation. In the event of an outbreak on campus, many people will be identified through contact tracing but under most circumstances (assuming proper physical distancing protocols are followed), only a fraction of those individuals will be infected. In some model runs, up to one-third of students needed to self-isolate following contact tracing, despite not being infected. 


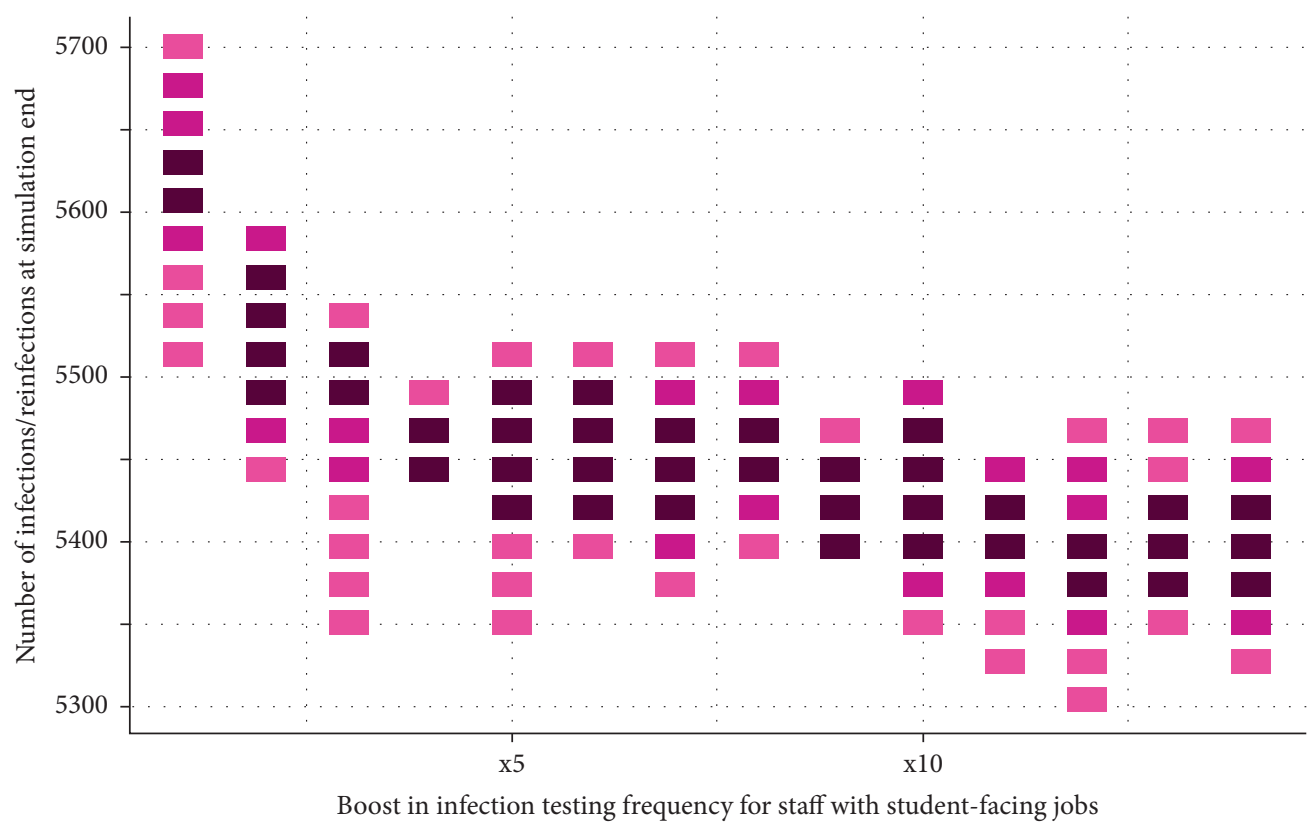

Figure 5: The number of infections (or reinfections) against the factor of testing frequency increases for staff with student-facing jobs. Numerous runs are binned and the color indicates clustering of runs, with darker colors corresponding to a greater number of runs.

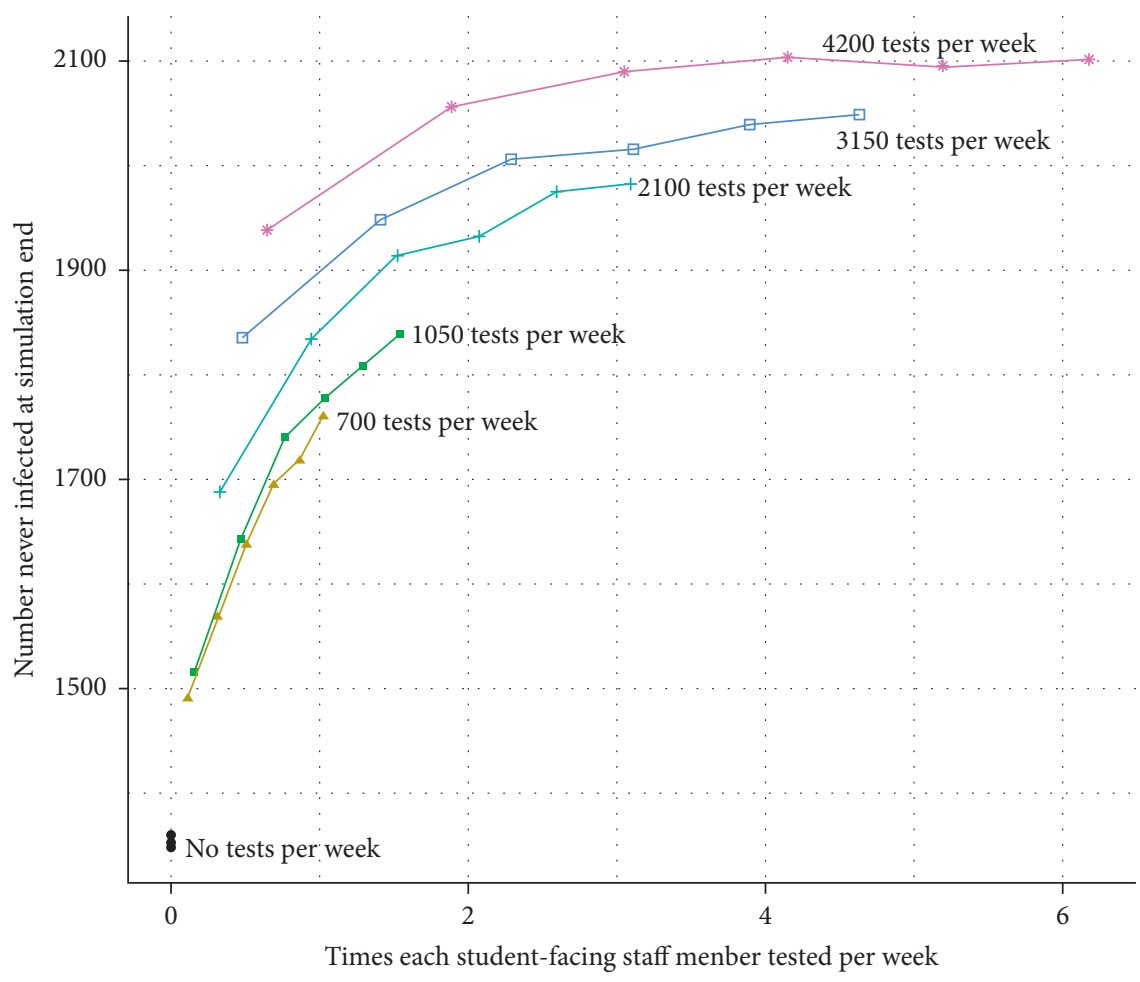

Figure 6: Health outcomes for six different testing configurations showing a trade-off between the weekly total number of tests and the testing frequency for student-facing staff. In this figure, each curve is a line of fixed cost, illustrating that better health outcomes can be achieved for lower cost, depending on how testing is performed.

This finding suggests that universities instituting rigorous contact tracing and isolation procedures will have to be ready for large numbers of students in isolation and will need to prepare critical systems accordingly. These include education about the need for self-isolation, food services, emotional support for people in quarantine (COVID-19 research already shows emotional factors associated with isolation and anxiety are critical for mental health, both acutely and long-term) and infrastructure to facilitate ongoing classroom participation remotely. 


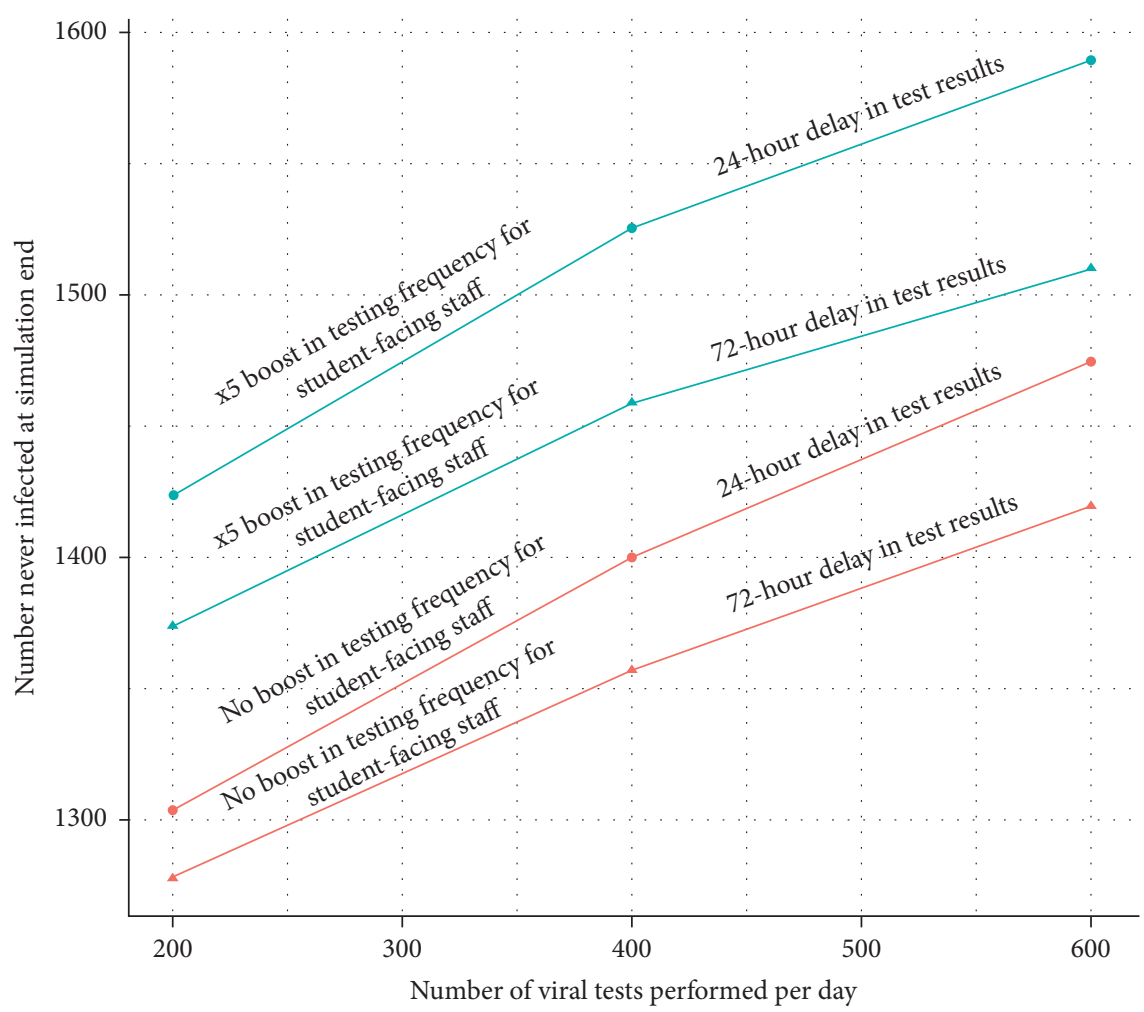

FIGURE 7: Health outcomes for three testing configurations showing the relative importance of increasing testing frequency for studentfacing staff and decreasing the delay in getting test results. In this figure, each curve is a line of fixed cost, illustrating that better health outcomes can be achieved for lower cost, depending on how testing is performed and how rapidly results are received and acted on.

Such support systems can be expensive, especially in personnel costs. Therefore, it is also important to keep in mind that traced isolation does not imply infection and that moving trace-flagged students to even more expensive dedicated housing is probably not cost-effective until symptoms appear or testing of self-isolated people shows an infection, at which point people can be moved to avoid spreading the virus through roommates and shared bathrooms.

3.7. Comparing Metrics. Metrics used to assess policy success reflect underlying values, which are critical human factors in a pandemic. To show that metrics matter, we ran a regression-subset analysis for the "minimize infections" metric (Figure 2) and also for the "hybrid" metric that tracks the difference between positive and negative outcomes (described above). Some factors are similarly important for both metrics:

High compliance with physical distancing

Hybrid (remote and in-person) classes

Other factors differ in importance:

Lengthening the history of contact tracing is more important for the hybrid metric

Boosting frequency of infection testing for studentfacing staff is significantly more important on the "minimize infections" metric
It follows that university administrators need to review their values carefully and select the most relevant metrics for their contexts in full awareness that alternative metrics would likely yield different findings for intervention effectiveness.

\section{Discussion and Conclusion}

Using TAU, we evaluate the possible effects of social distancing, contact tracing, testing, activity closures, dedensifying strategies, and a variety of other interventions. TAU shows that social-distancing requirements (including maskwearing) have the most significant effect on infections, followed by central tracking and boosting testing frequency for critical networks nodes, which include staff with intensive student contact. However, high compliance is needed for optimal effect. For social distancing, we see a tipping point of effectiveness around a compliance rate of $60 \%$, showing the need to create "buy in" among students, staff, and faculty, which calls for targeted publicity campaigns.

The use of a variety of metrics allows us to take different viewpoints into account, promoting a multivalue perspective in which alternatives can be compared and side effects identified. Such multivalue perspectives contest the tendency to focus on one or two domains that capture immediate attention, to the exclusion of others.

We would like to validate TAU against real-world data from universities. Unfortunately, such datasets do not yet exist, though TAU's finding about the importance of 
compliance has been amply confirmed by the fact that the numerous university closures in September 2020 were directly attributable to compliance failures. The New York Times data on universities from September 2020 onwards only report infections and test results and supply no data on policies in place, or on compliance, so cannot be employed to validate TAU.

It is worth thinking further about the role of computational simulations in the absence of the complete realworld datasets needed for comprehensive validation. As noted earlier, even without validation against relevant university data, TAU's architecture, from parameters to processes, are themselves well grounded, so TAU is useful as a way to think deeply into the problem of university management under pandemic conditions, detecting critical explanatory factors and tipping points for intervention effectiveness. This point has been made in a series of important publications, beginning with Troitzsch [4]. More recent practical recommendations for validation can be found in Davis et al. [5], which directly addresses the meaning of simulations like TAU, which are well validated at the low level of causal architecture but cannot be validated at the high level for want of quantitative data of the right kinds. The same point is explored in depth by Saldanha et al. [6].

TAU has limits, which we think of as opportunities for extension. For example, we aim to integrate TAU with county-level data from the COVID-19 Health Care Coalition's dashboard (c19hcc.org) to introduce greater realism in the way TAU handles the porousness of university campuses. We would want to add real-time calibration against university infection statistics. A formal cost-benefit analysis module and an equity-and-justice metric are currently under development for inclusion in the simulation. Additionally, enriching the dashboard at http://mindandculture.org/projects/modelingsocial-systems/vivid/vivid-dashboard/ would simplify the threefold process of configuring TAU, running analyses on TAU-simulated data, and generating visualizations to help communicate policies. There are also a few variations on interventions already included that might prove useful for some universities, such as more intensive contact tracing that attempts to locate superspreaders. In June 2020, we released TAU as an open-source product to allow others to make such adjustments and thereby contribute to the project of helping colleges and universities manage the pandemic.

Despite these limitations, TAU is already a powerful decision support tool for universities. It demonstrates that an artificial university-implemented as an agent-based model using contact networks, integrating an epidemiological model with sensitivity to human factors, and calibrated against publicly available data, following the guidelines of Diallo et al. [7] — can generate valuable insights into the challenge of operating universities under pandemic conditions.

\section{Data Availability}

A dashboard interface for exploring TAU is available at http:// mindandculture.org/projects/modeling-social-systems/vivid/ vivid-dashboard/. This site includes links to the model, documentation, and data (see https:/github.com/ centerformindandculture/TheArtificialUniversity).

\section{Disclosure}

The work presented was collaboratively conducted under the COVID-19 Healthcare Coalition. The views, opinions, and/or findings contained in this paper provided by the author are those of The MITRE Corporation and should not be construed as an official government position, or decision, unless designated by other documents. The contributions are approved for public release, distribution unlimited (Case-Nr. 20-017897. ( 2020 The MITRE Corporation, all rights reserved).

\section{Conflicts of Interest}

The authors declare that they have no conflicts of interest.

\section{Acknowledgments}

The authors are pleased to acknowledge the work of Dr. Eric Weisel, Executive Director of the Virginia Modeling, Analysis \& Simulation Center at Old Dominion University, and some of his staff for their provision of a dashboard interface to support nonprogrammatic access to the simulated data from The Artificial University. This research was funded by the organizations employing the authors: Center for Mind and Culture, Boston University, Virginia Modeling, Analysis \& Simulation Center at Old Dominion University, Western Sydney University, and The MITRE Corporation.

\section{References}

[1] P. T. Gressman and J. R. Peck, "Simulating COVID-19 in a university environment," Mathematical Bioscience, vol. 328, Article ID 108436, 2020.

[2] J. H. Kwakkel and P. Erik, "Exploratory modeling and analysis, an approach for model-based foresight under deep uncertainty," Technological Forecasting and Social Change, vol. 80, no. 3, pp. 419-431, 2013.

[3] A. F. Siegenfeld, N. N. Taleb, and Y. Bar-Yam, "What models can and cannot tell us about COVID-19," Proceedings of the National Academy of Sciences, vol. 117, no. 28, 2020.

[4] K. G. Troitzsch, "Validating simulation models," in Proceedings of the 18th European Simulation Multiconference: Networked Simulations and Simulation Networks, Magdeburg, Germany, June 2004.

[5] P. K. Davis, A. O’Mahony, T. R. Gulden, O. A. Osoba, and K. Sieck, "Priority challenges for social and behavioral research and its modeling," Santa Monica, United States: RAND Corporation, 2018.

[6] E. Saldanha, L. M. Blaha, A. V. Sathanur, H. Nathan, S. Volkova, and M. Greaves, "Evaluation and validation approaches for simulation of social behavior: challenges and opportunities," in Social-Behavioral Modeling for Complex Systems, N. J. Hoboken, Ed., John Wiley \& Sons, New Jersey, NY, USA, pp. 495-519, 2019.

[7] S. Y. Diallo, W. J. Wildman, F. L. Shults, and A. Tolk, "Human simulation: perspectives, insights, and applications," in New Approaches to the Scientific Study of Religion Series, Springer, Berlin, Germany, 2019. 